\title{
Distribution of human Cytomegalovirus gB genotypes in samples from pediatric patients in Parma during the period I 995-2003
}

\author{
Maria Cristina Arcangeletti, Federica Motta, Isabella Rodighiero, Diego Germini, \\ Giuseppe Dettori, Carlo Chezzi \\ Unità Operativa diVirologia. Dipartimento di Patologia e Medicina di Laboratorio.Azienda Ospedaliero-Universitaria di Parma
}

Key words: Cytomegalovirus, Glycoprotein B, Pediatric population, RFLP

Distribuzione dei genotipi gB di citomegalovirus umano in campioni appartenenti a pazienti pediatrici nell'area di Parma nel periodo I995-2003

\section{SUMMARY}

Background. Human Cytomegalovirus (HCMV) infection is a leading cause of developmental disability and late neurological sequelae in children. Several literature data indicate that HCMV pericapsidic glycoprotein $B(g B)$ is highly immunogenic and is involved in virus-cell interaction. The gB gene has hypervariable regions producing four genotypes (gBI, gB2, gB3, gB4); however, the correlation between gB genotypes and HCMV infection outcome remains unclear.

Objectives. The main goal of this study was that of evaluating the distribution of HCMV gB genotypes in samples from pediatric population in Parma with congenital, perinatal or post-natal infections, in order to find a correlation between viral gB genotypes and the clinical outcome of the infection.

Study design. Forty eight urine samples, selected between 1999 and 2003 and stored at $-80^{\circ} \mathrm{C}$, underwent DNA extraction, nested PCR amplification of a gB gene region and restriction polymorphism analysis (RFLP).

Results. The gB genotypes distribution in the considered pediatric population was as follows: gBI was the most diffused (45.83\%) followed by gB2 (22.92\%), gB3 (16.67\%) and gB4 (I4.58\%).

Conclusions. In the considered population, gBI was the most represented genotype and was often found in congenital and perinatal symptomatic infections, as well as in post-natal, asymptomatic infections.

\section{INTRODUZIONE}

Cytomegalovirus umano (HCMV) viene ad oggi considerato tra gli agenti eziologici più importanti nell'indurre anomalie congenite e/o sequele neurologiche tardive nel bambino, a seguito di infezione materna primaria (1). Diversamente da altre infezioni virali contratte durante il periodo di gestazione, la presenza di anticorpi materni nel caso di citomegalovirus, non garantisce la completa protezione del feto: ne consegue che, sebbene la trasmissione materno-fetale di HCMV interessi, nella maggioranza dei casi, bambini nati da madri che contraggono un'infezione primaria durante la gravidanza, sequele tardive possono manifestarsi anche nei nati da madri con riattivazione dell'infezione (8). La diagnosi di laboratorio prenatale di infezione congenita da HCMV viene, di norma, eseguita su liquido amniotico mediante PCR quantitativa, in grado di discriminare con accuratezza tra feti infettati e non infettati $(3,6)$.

Inoltre, è da tenere in dovuta considerazione il fatto che l'infezione da HCMV possa avvenire anche subito dopo la nascita, in particolare attraverso la saliva o il latte materno (infezione perinatale), $\mathrm{o}$ in epoca più tardiva (infezione post-natale), con esiti normalmente più favorevoli rispetto all'infezione congenita; anche in questi casi, la diagnosi di laboratorio mediante metodi molecolari o convenzionali è in grado di attestare la presenza del virus.

Tenendo conto dei diversi esiti che può avere un'infezione da HCMV, da quella inapparente a una vasta gamma di manifestazioni patologiche, è sentita l'esigenza di individuare strumenti in grado di fornire informazioni utili circa le possibili ricadute cliniche dell'infezione da parte di tale agente virale.

Numerosi dati di letteratura sottolineano come la glicoproteina pericapsidica B (gB) di HCMV, coinvolta nell'interazione con il recettore cellulare e bersaglio per gli anticorpi neutralizzanti, rappresenti un potenziale fattore di virulenza per tale agente $(5,7,9,10,11,12,15)$. Questo componente virale presenta regioni ipervariabili che rendono ragione della diversità genetica di $\mathrm{gB}$ riscon-

\section{Corresponding author: Maria Cristina Arcangeletti}

Microbiology Section, Department of Pathology and Laboratory Medicine, University of Parma

Viale A. Gramsci, I4 - 43I26 Parma, Italy - Tel.: +39 052। 033499 - Fax: +39 052I 993620

E-mail: mariacristina.arcangeletti@unipr.it 
trata nei differenti ceppi virali circolanti (2). Sulla base di tale variabilità gli stipiti di HCMV possono essere classificati in quattro genotipi $\mathrm{gB}$ mediante l'analisi dei frammenti genici ottenuti dopo taglio enzimatico (RFLP: "Restriction fragment length polymorphism").

Studi recenti supportano l'ipotesi di una correlazione tra genotipo $\mathrm{gB}$ e quadri clinici di diversa gravità per differenti categorie di individui, quali soggetti adulti sottoposti a trapianto di midollo, pazienti con AIDS e soggetti pediatrici $(5,7,10$, 11, 12).

Questo studio si è proposto di valutare la distribuzione dei diversi genotipi gB di HCMV nell'ambito di un campione di popolazione pediatrica nell'area di Parma e di verificare l'esistenza di una eventuale correlazione tra genotipo virale e presenza di quadri clinici riferibili ad infezione da HCMV, in particolare in neonati con presunta infezione congenita.

\section{MATERIALI E METODI}

Campioni clinici. Sono stati inclusi nello studio 48 ceppi virali di isolamento clinico, ottenuti da campioni di urina di pazienti pediatrici (età compresa tra 1 giorno di vita e 6 anni) con infezione da HCMV, inviati presso l'Unità Operativa di Virologia dell'Azienda Ospedaliero-Universitaria di Parma nel periodo gennaio 1999-dicembre 2003; per tali pazienti è stato possibile analizzare i dati contenuti nelle cartelle cliniche, con particolare riguardo agli esiti immediati e/o tardivi dell'infezione da HCMV.

Colture cellulari. Fibroblasti di polmone embrionale umano MRC5 sono stati coltivati in monostrato in terreno di coltura "Minimum Essential Medium" (MEM), modificato con sali di Earle ed addizionato di siero fetale di vitello (10\%), sodio piruvato $(1 \%)$, amminoacidi non essenziali (1\%), L-glutammina (1\%) ed antibiotici (penicillina 10.000 unità $/ \mathrm{ml}$ e streptomicina $10.000 \mathrm{mg} / \mathrm{ml}$ ) (ditta PAA). Tali cellule sono state utilizzate per la coltivazione dei ceppi di HCMV di controllo e per quelli di isolamento clinico.

Ceppi virali di controllo. I ceppi virali scelti come controllo sono rappresentati dagli stipiti umani AD169 (ATCC n. VR-538) e Towne (ATCC n. VR-977) di HCMV, riprodotti in cellule MRC5 e titolati, sulla base della determinazione quantitativa dell'infettività, mediante una reazione di immunofluorescenza condotta sulle stesse cellule, impiegando un anticorpo monoclonale che riconosce un epitopo comune alle 2 proteine precocissime maggiori (IEp72/IEp86) di citomegalovirus.

Co-coltivazione di fibroblasti embrionali umani MRC5 con cellule infettate con HCMV.
I monostrati di cellule MRC5, previamente inoculati con i campioni biologici di interesse, sono stati raccolti in presenza di un evidente effetto citopatico caratteristico di HCMV e conservati a $80^{\circ} \mathrm{C}$. In seguito, le suddette cellule infettate sono state scongelate ed utilizzate per la co-coltivazione con cellule MRC5 non infettate, al fine di ottenere un arricchimento in particelle virali.

Purificazione del DNA. I campioni, rappresentati da cellule MRC5 infettate con i ceppi virali di controllo o con i ceppi virali di isolamento clinico (previa co-coltivazione per aumentare la concentrazione virale, come precedentemente descritto), sono stati privati del terreno di mantenimento e raccolti mediante tripsinizzazione; dopo due lavaggi, i sedimenti cellulari sono stati sottoposti a purificazione del DNA utilizzando il sistema "Elute gene mammalian Kit" (ditta SigmaAldrich), seguendo le indicazioni della ditta produttrice.

Amplificazione del gene gB mediante nestedPCR. Il DNA estratto dai 48 ceppi di HCMV di isolamento clinico è stato amplificato mediante nested-PCR ("primers" $g B$ esterni:

5'-GGAAACGTGTCCGTCTT- 3'; 5'-GAGTAGCAGCGTCCTGGCGA - 3'; "primers" $g B$ interni: 5'- TGGAACTGGAACGTTTGGC-3'; 5' -GAAACGCGCGGCAATCGG- 3').

Le reazioni di amplificazione sono state condotte per mezzo di un termociclatore automatico (Eppendorf Mastercycler Gradient); i prodotti di amplificazione sono stati rivelati mediante migrazione elettroforetica in gel d'agarosio all'1\% addizionato di bromuro di etidio (Sigma-Aldrich).

\section{Digestione enzimatica.}

Gli amplificati ottenuti (302 pb) sono stati sottoposti a digestione enzimatica utilizzando gli enzimi RSA I (Rhodopseudomonas sphaeroides) e Hinf I (Haemophilus influenzae) [ditta Fermentas] ed incubati a $37^{\circ} \mathrm{C}$ per circa 16 ore; al termine del periodo di incubazione, i prodotti sono stati rivelati mediante migrazione elettroforetica in gel d'agarosio al $2 \%$.

Il profilo di migrazione dei diversi frammenti di DNA e la loro lunghezza sono stati analizzati per confronto con un adatto sistema di riferimento a peso molecolare noto $(1 \mathrm{~kb}$ plus ladder: $100 \mathrm{pb}-$ $12000 \mathrm{pb}$; ditta Invitrogen).

Criteri di suddivisione del campione di popolazione pediatrica in gruppi in relazione alle diverse tipologie di infezione da HCMV. Sulla base dei dati contenuti nelle cartelle cliniche dei pazienti considerati nello studio, è stato possibile suddividere il campione di popolazione in: soggetti con presunta infezione congenita (18 soggetti), con presunta infezione perinatale (14 soggetti) e con presunta infezione post-natale (16 soggetti). 
- Pazienti con presunta infezione congenita:

- Per 11 pazienti è stato possibile reperire il dato relativo alla sieroconversione materna durante il periodo di gestazione;

- Due pazienti sono risultati positivi alla Guthrie card (screening neonatale obbligatorio per legge);

- Per 4 pazienti non è stato possibile desumere dati relativi alla sieroconversione materna in epoca gestazionale; tuttavia, in 2 casi l'urina del neonato è risultata positiva alla nascita ed in altri 2 casi la positività per HCMV in campioni di urina è stata riscontrata tra la terza e la quarta settimana di vita.

- Pazienti con presunta infezione perinatale: 14 pazienti con età compresa tra 1 e 4 mesi di vita, per $\mathrm{i}$ quali era anche disponibile l'informazione relativa all'allattamento al seno.

- Pazienti con presunta infezione post-natale: 16 pazienti con intervallo di età compreso tra 5 mesi e 6 anni.

\section{RISULTATI}

Analisi elettroforetica del profilo di restrizione della glicoproteina b di ceppi di HCMV isolati da campioni di urina inclusi nello studio.

L'analisi elettroforetica del polimorfismo di restrizione dei genotipi $\mathrm{gB}$ degli stipiti di HCMV isolati da 48 campioni di urina pervenuti all'Unità Operativa di Virologia dell'Azienda Ospedaliero-Universitaria di Parma nell'arco temporale di 5 anni (19992003), ha messo in evidenza, nel campione di popolazione considerato, la presenza di tutti e 4 i genotipi virali (Figura I).

Distribuzione dei genotipi $\mathrm{gB}$ di ceppi di HCMV isolati da urina nel campione di popolazione pediatrica oggetto dello studio.

Per quel che riguarda la distribuzione dei genotipi gB di HCMV identificati nell'ambito dei 48 soggetti allo studio, $\mathrm{gB} 1$ è stato riscontrato in 22 pazienti $(45.83 \%)$; dimostrandosi prevalente nella popolazione pediainfezione. trica considerata; i genotipi gB2, gB3 e gB4 sono stati, invece, evidenziati in $11(22.92 \%), 8$ $(16.67 \%)$ e $7(14.58 \%)$ pazienti, rispettivamente.

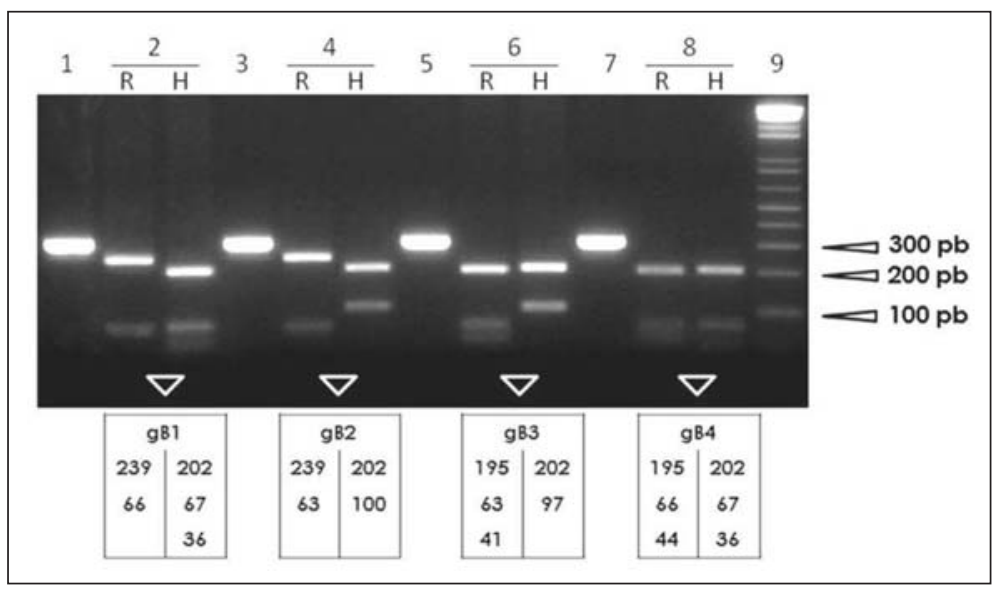

Figura I. Esempio di analisi elettroforetica del profilo di restrizione della glicoproteina B di ceppi di HCMV isolati da campioni di urina. Pozzetti I, 3, 5, 7: prodotti di amplificazione ottenuti mediante nested-PCR utilizzando come stampo DNA purificato da cellule MRC5 co-coltivate con ceppi virali di isolamento clinico. Pozzetti $2 \mathrm{R}-2 \mathrm{H}, 4 \mathrm{R}-4 \mathrm{H}, 6 \mathrm{R}-6 \mathrm{H}, 8 \mathrm{R}-8 \mathrm{H}$ : frammenti ottenuti dalla digestione dei prodotti di amplificazione del gene $g B$ con gli enzimi di restrizione Rsa I e Hinf l, rispettivamente. La dimensione dei prodotti di digestione è riportata nella parte inferiore della figura. Pozzetto 9: pesi molecolari noti (100pb-I 2000pb).

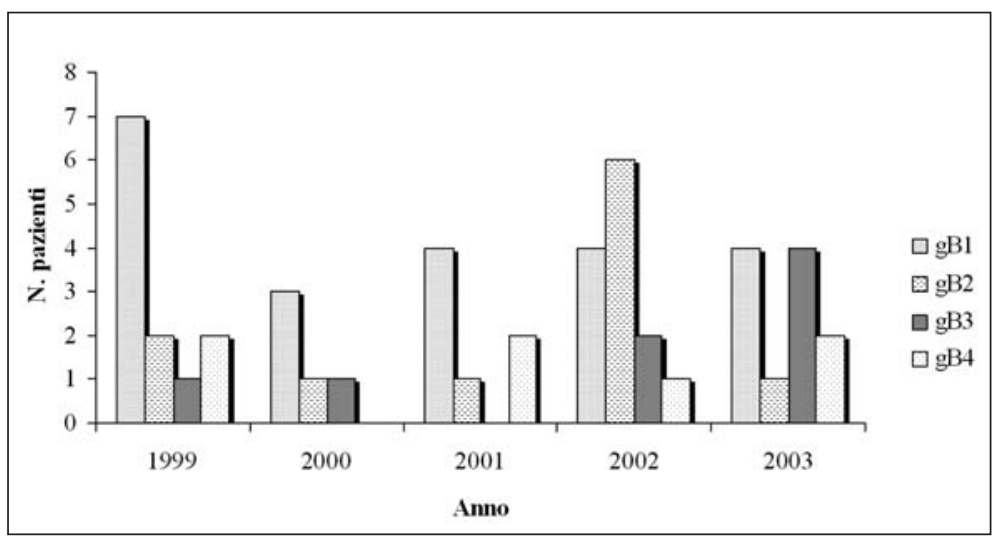

Figura II. Distribuzione annuale dei genotipi gB di HCMV nel campione di popolazione pediatrica considerato.

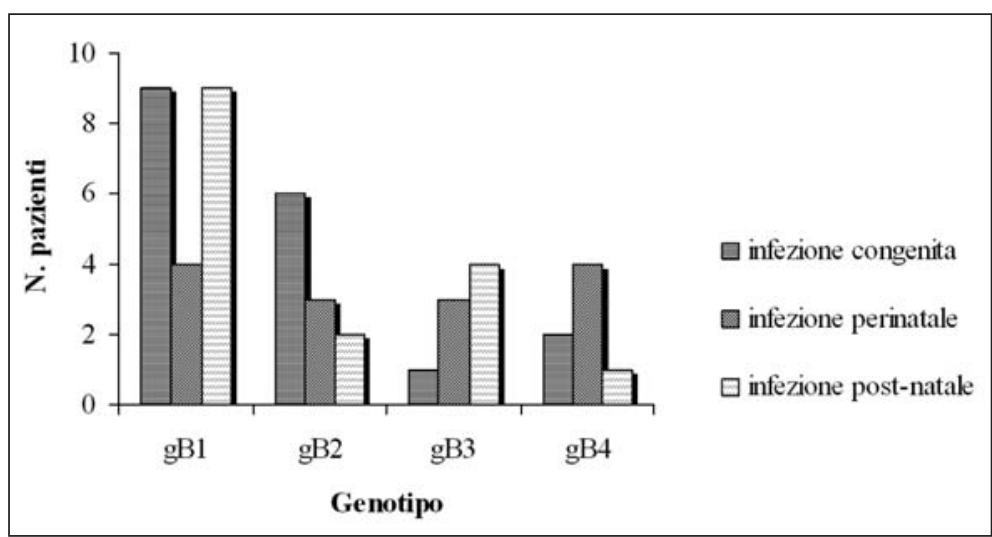

Figura III. Distribuzione dei genotipi gB identificati per tipologia di 
L'analisi della distribuzione annuale dei genotipi gB di HCMV (Figura II) ha mostrato, in generale, la prevalenza del genotipo gB1 nella maggior parte degli anni studiati. Tuttavia, è possibile notare come, considerando gli anni con numero maggiore e pressoché equivalente di campioni analizzati (1999, 2002, 2003), si osservi una certa variabilità di distribuzione. Nello specifico, il genotipo gB1 è risultato francamente predominante nel 1999, mentre il genotipo gB2 lo è stato nel 2002. Nel 2003, i genotipi gB1 e gB2 sono stati parimenti presenti.

Considerando la distribuzione dei genotipi $\mathrm{gB}$ nelle tre tipologie di infezione da HCMV (congenita, perinatale e post-natale) prese in esame, indipendentemente dalla presenza o assenza di sintomatologia significativa (Figura III), si nota come il genotipo $\mathrm{gB} 1$ prevalga nelle infezioni congenite e post-natali, il gB2 nelle infezioni congenite (anche se in misura minore rispetto a $\mathrm{gB} 1)$, il gB3 nelle infezioni perinatali e post-natali ed il gB4 nelle infezioni perinatali.

Distribuzione dei genotipi $\mathrm{gB}$ identificati per tipologia di infezione ed in base alla presenza 0 all'assenza di sintomatologia.

Dall'analisi dei dati riportati nella Tabella 1 emerge come, mentre la distribuzione dei genotipi $\mathrm{gB}$ nei soggetti asintomatici sembra essere equamente ripartita tra $\mathrm{gB} 1, \mathrm{gB} 2$, $\mathrm{gB} 3$ e gB4, la situazione è nettamente diversa in presenza di sintomatologia riferibile ad infezione da HCMV. In questi casi, infatti, si osserva una predominanza del genotipo gB1 sugli altri tre. In particola- re, è da notare come, nei pazienti con sintomi a carico del sistema nervoso centrale (lesione retinica, calcificazioni intracraniche, aumento del volume ventricolare, ipoacusia grave) sia presente esclusivamente il genotipo gB1. Tali informazioni sono state rielaborate ed espresse in forma grafica, considerando il tipo di infezione (congenita, perinatale, post-natale) e la presenza o l'assenza di sintomatologia (Figura IV).

In particolare, la distribuzione dei genotipi $\mathrm{gB}$ nell'ambito delle infezioni congenite mostra come

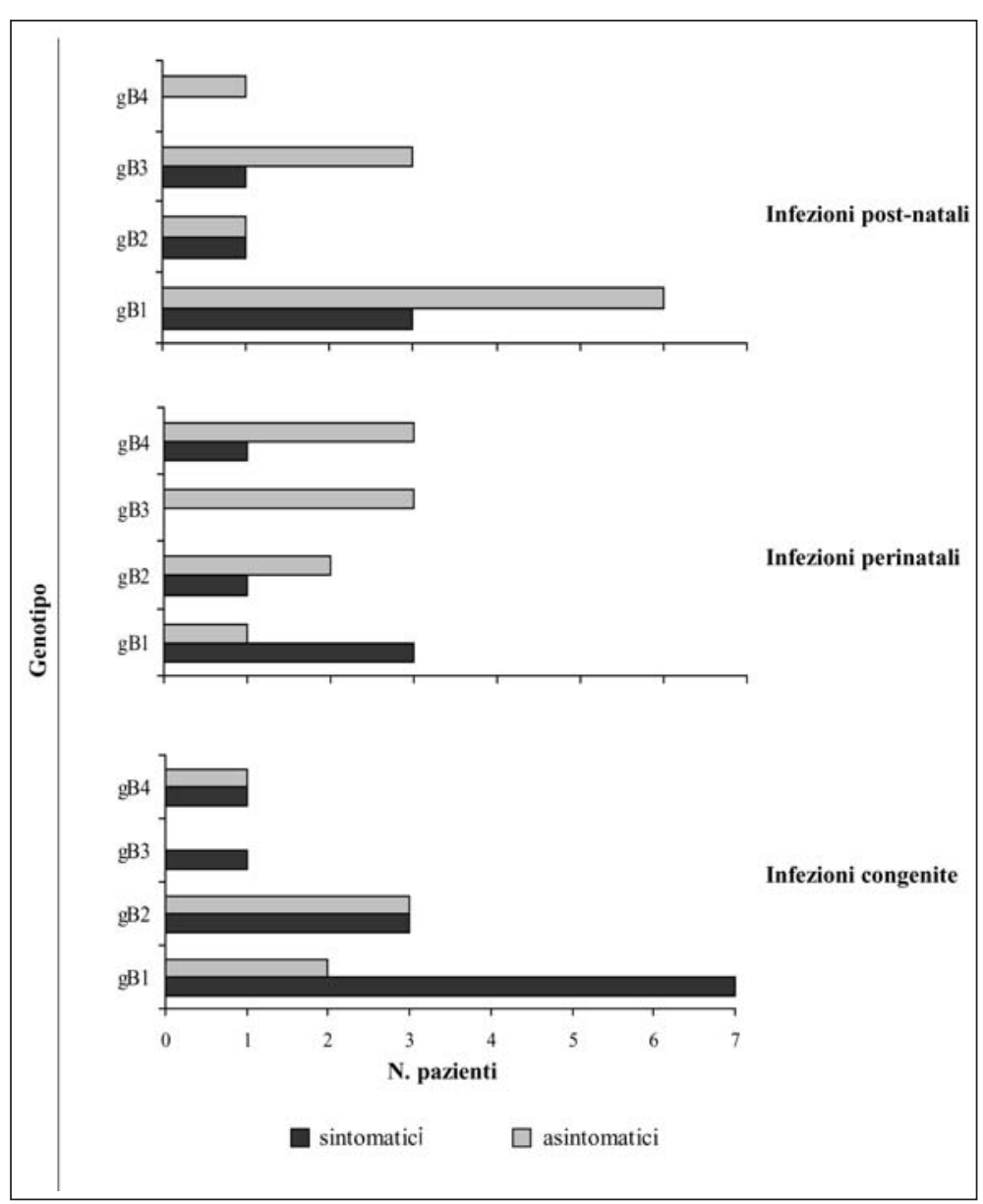

Figura IV. Distribuzione dei genotipi gB nelle infezioni congenite, perinatali e post-natali.

Tabella I. Distribuzione dei genotipi gB in base alla sintomatologia, nel campione di popolazione pediatrica considerato.

\begin{tabular}{|c|c|c|c|c|c|}
\hline & gB I & gB2 & gB3 & gB4 & TOTALE \\
\hline TOTALE PAZIENTI: & 22 & 11 & 8 & 7 & 48 \\
\hline ASINTOMATICI & 9 & 6 & 6 & 5 & 26 \\
\hline SINTOMATICI & 13 & 5 & 2 & 2 & 22 \\
\hline - ittero & $\mathrm{I}$ & $\mathrm{I}$ & 0 & 0 & 2 \\
\hline - aumento dei livelli di transaminasi & 4 & 4 & $\mathrm{I}$ & $\mathrm{I}$ & 10 \\
\hline - aumento dei livelli di transaminasi e del volume epatico & 2 & 0 & 0 & 0 & 2 \\
\hline - aumento dei livelli di transaminasi e del volume splenico & I & 0 & 0 & 1 & 2 \\
\hline - aumento dei livelli di transaminasi e epatosplenomegalia & 0 & 0 & $\mathrm{I}$ & 0 & $\mathrm{I}$ \\
\hline - aumento dei livelli di transaminasi e lesione retinica & 2 & 0 & 0 & 0 & 2 \\
\hline - aumento dei livelli di transaminasi e calcificazioni intracraniche & I & 0 & 0 & 0 & 1 \\
\hline - calcificazioni intracraniche, aumento del volume ventricolare, cataratta & $\mathrm{I}$ & 0 & 0 & 0 & $\mathrm{I}$ \\
\hline - calcificazioni intracraniche e ipoacusia grave & I & 0 & 0 & 0 & $\mathrm{I}$ \\
\hline
\end{tabular}


la maggior parte delle infezioni sintomatiche sia riferibile al genotipo $\mathrm{gB} 1$. Il genotipo $\mathrm{gB} 2$ sembra invece equamente ripartito tra le infezioni congenite sintomatiche e asintomatiche, mentre i genotipi gB3 e gB4 sembrano essere scarsamente rappresentati in questa categoria.

Per quanto riguarda le infezioni perinatali, gB1 prevale in quelle sintomatiche, come già evidenziato per le infezioni congenite. Gli altri tre genotipi si distribuiscono prioritariamente nelle infezioni asintomatiche.

L'analisi della distribuzione dei genotipi gB nell'ambito delle infezioni post-natali mostra sempre una netta prevalenza del genotipo gB1, anche se, differentemente da quanto osservato per le infezioni congenite, esso è più frequentemente associato ad infezioni asintomatiche.

\section{DISCUSSIONE}

L'analisi dei risultati ha dimostrato, nel campione di popolazione pediatrica considerato, la presenza di tutti e quattro i principali genotipi $\mathrm{gB}$ di HCMV, con una netta prevalenza del genotipo $\mathrm{gB} 1$; tale dato sembra essere in accordo con quanto rilevato in altre realtà geografiche $(4,5,10,11$, $13,14)$. In particolare, analizzando separatamente le tre categorie di infezione (congenita, perinatale, post-natale) sulla base della presenza o dell'assenza di sintomatologia significativa, i risultati ottenuti supportano una prevalenza del genotipo gB1 in soggetti sintomatici sia in caso di infezione congenita, sia in caso di infezione perinatale, come riportato anche da altri Autori $(5,10,11$, 14), mentre la tendenza si inverte nel gruppo delle infezioni post-natali, in cui gli stipiti virali gB1 sarebbero principalmente responsabili di infezioni asintomatiche.

$\mathrm{Se}$, da un lato, i dati sulle infezioni congenite e perinatali supportano un legame tra genotipo gB1 ed una maggiore virulenza di tali stipiti virali, dall'altro la prevalenza di gB1 anche nelle infezioni post-natali asintomatiche fa riflettere su come, verosimilmente, numerosi fattori entrino in gioco nel determinare i diversi esiti dell'infezione, non ultimo l'immaturità immunologica fetale.

I genotipi $\mathrm{gB} 3 \mathrm{e} g \mathrm{~g} 4$, poco rappresentati nel gruppo di infezioni congenite considerato, sembrano essere legati prevalentemente ad infezioni asintomatiche nelle categorie di infezioni perinatali e post-natali.

E interessante notare che, per quel che riguarda $\mathrm{gB} 4$, i risultati ottenuti in questo lavoro scientifico si discostano dai dati epidemiologici riscontra$\mathrm{ti}$ in altre aree, dove tale genotipo non viene mai rilevato $(13,14)$.

In conclusione, questo studio offre spunti interessanti su aspetti dell'infezione da HCMV e su fat- tori virali implicati nel modulare una differente virulenza degli stipiti coinvolti e nel contribuire potenzialmente alla diversa gravità della malattia. Tuttavia, bisogna anche tenere presente che le dimensioni numericamente ridotte del campione di popolazione considerato non permettono di dare una valenza statisticamente significativa ai dati riscontrati, sebbene, come già accennato, questi siano in linea con quanto pubblicato in letteratura, in particolare per quanto attiene a $\mathrm{gB} 1(5,10$, 11, 14).

Ciò nonostante, tale indagine retrospettiva può essere considerata come uno studio pilota da potere applicare sistematicamente ed in tempo reale in futuro, al fine di stabilire un osservatorio epidemiologico molecolare che dia indicazioni utili sulla circolazione dei diversi genotipi $\mathrm{gB}$ di HCMV nell'area di Parma.

\section{BIBLIOGRAFIA}

1. Boppana SB, Rivera LB, Fowler KB, Mach M, Britt WJ. Intrauterine transmission of Cytomegalovirus to infants of women with preconceptional immunity. $N$ Engl J Med 2001; 344: 1366-71.

2. Chou SW, Dennison KM. Analysis of interstrain variation in Cytomegalovirus glycoprotein B sequences encoding neutralization-related epitopes. $J$ Infect Dis 1991; 163: 1229-34.

3. Ducroux A, Cherid S, Benachi A, Ville Y, Leurez-Ville M. Evaluation of new commercial real-time PCR quantification assay for prenatal diagnosis of Cytomegalovirus congenital infection. $J$ Clin Microbiol 2008; 46: 2078-80.

4. Fan J, Zhang X, Chen XM, et al. Monitoring of human Cytomegalovirus glycoprotein B genotypes using real-time quantitative PCR in immunocompromised Chinese patients. J Virol Methods 2009; 160: 74-7.

5. Jin H, Wang X, Li S. Human Cytomegalovirus glycoprotein B genotype correlates with different symptoms of infected infants. Intervirology 2007; 50: 21923.

6. Lazzarotto T, Guerra B, Lanari M, Gabrielli L, Landini MP. New advances in the diagnosis of congenital Cytomegalovirus infection. J Clin Virol 2008; 41: 192-7.

7. Rasmussen L, Hong C, Zipeto D, et al. Cytomegalovirus gB genotype distribution differs in human immunodeficiency virus-infected patients and immunocompromised allograft recipients. J Infect Dis 1997; 175: 179-84.

8. Rousseau T, Douvier S, Reynaud I, et al. Severe fetal cytomegalic inclusion disease after documented maternal reactivation of Cytomegalovirus infection during pregnancy. Prenat Diagn 2000; 20: 333-6.

9. Tanaka K, Numazaki K, Tsutsumi H. Human Cytomegalovirus genetic variability in strains isolated from Japanese children. J Med Virol 2005; 76: 356-60.

10. Terabe K, Sugiyama K, Goto K, et al. Relationship between human Cytomegalovirus glycoprotein B genotype and serum alanine amonitransferase elevation in infants. Tohoku J Exp Med 2004; 203: 339-44.

11. Trincado DE, Scott GM, White PA, Hunt C, Rasmussen L, Rawlinson WD. Human Cytomegalovirus strains associated with congenital and perinatal infections. J Med Virol 2000; 61: 481-7. 
12. Wu X, Wang Y, Xu Y, et al. Cytomegalovirus glycoprotein $\mathrm{B}$ genotype in hematopoietic stem cell transplant patients from China. Biol Blood Marrow $\mathrm{Tr}$ 2010; 16: 647-52.

13. Yamamoto AJ, Mussi-Pinhata MM, de Deus Wagatsuma VM, Marin LJ, Duarte G, Figuiredo LTM. Human Cytomegalovirus glicoprotein B genotypes in Brazilian mothers and their congenitally infected infants. J Med Virol 2007; 79: 1164-8.
14. Yu ZS, Zou CC, Zheng JY, Zhao ZY. Cytomegalovirus $\mathrm{gB}$ genotypes and clinical features in chinese infants with congenital infections. Intervirology 2006; 49: 281-5.

15. Zipeto D, Hong C, Gerna G, et al. Geographic and demographic differences in the frequency of human cytomegalovirus gB genotypes 1-4 in immunocompromised patients. Aids Res Hum Retroviruses 1998; 14: 533-6. 